\title{
Throughput Analysis and Enhancement for CSMA Based Wireless Networks
}

\author{
Younggoo Kwon \\ Konkuk University, 1 Hwayang-dong, Kwangjin-gu, Seoul, 143-701, Korea \\ ygkwon@konkuk.ac.kr
}

\begin{abstract}
We studied the performance of contention based medium access control (MAC) protocols. We used a novel technique for estimating the throughput, and other parameters of interest, of such protocols. In this paper, a new assumption for the theoretical throughput limit in the distributed CSMA based MAC algorithm is introduced. Through the performance analysis and simulation studies, the proposed algorithm shows significant performance improvements in CSMA based wireless networks.
\end{abstract}

\section{Introduction}

In many performance analysis papers for the binary exponential backoff based CSMA algorithms, the performance analysis starts from the assumption that all stations have the same average contention window range in steady state [1]-[3]. The same average contention window range for all active stations can be understood that all stations have the same average probability of packet transmission in steady state. The performance of many binary exponential backoff based MAC algorithms, including the IEEE 802.11 MAC algorithm, can be explained well by using the assumption that all stations have the same average contention window range in steady state. Furthermore, the optimum value which will minimize the wasting overheads during the contention procedure can be derived for a given number of active stations [1]-[3]. However, there are still the wasting overheads come from the inherent limitation of the assumption that all stations have the same contention window range for packet transmission in steady state.

\section{Enhanced CSMA}

Under high traffic load and under some ergodicity assumption, we can obtain the simplified expression for the throughput:

$$
\rho=\frac{\bar{m}}{E\left[N_{c}\right]\left(E\left[B_{c}\right] \cdot t_{s}+\bar{m}+D I F S\right)+\left(E\left[B_{c}\right] \cdot t_{s}+\bar{m}+S I F S+A C K+D I F S\right)}
$$

where $E\left[N_{c}\right]$ is the average number of collisions, $E\left[B_{c}\right]$ is the average number of idle slots. 
From this result, we can see that the theoretical throughput limit would be the following: a successful packet transmission must be followed by another successful packet transmission without any overheads, in which case, $E\left[N_{c}\right]=0, E\left[B_{c}\right]=0$.

If we could develop a contention-based MAC algorithm, which assigns a backoff timer 0 to the station in transmission while assigns all other stations' backoff timers to $\infty$ for each contention cycle, then we could achieve the perfect scheduling, leading to the theoretical throughput limit. Unfortunately, such a contention-based MAC algorithm does not exist in practice. However, this does provide us the basic idea how to improve the throughput performance in the MAC algorithm design. We can use the operational characteristics of the perfect scheduling to design more efficient contention-based MAC algorithm. One way to do so is to design a MAC protocol to approximate the behavior of perfect scheduling. To achieve the similar operational characteristics of perfect scheduling, the proposed MAC algorithm provides the following design factors: Large contention window range \& small idle slots, long-term fairness, backoff timer realignment.

\section{Performance Evaluations}

We consider two kinds of contention window sizes, one for the whole contention procedure including deferring conditions, $E[C W]$, and the other for the case of transmitting a packet, $E[C W]_{P k S e n d}$. The relation between the average contention window size for each contention procedure $E[C W]$ and the probability of a successful packet transmission for one station $p_{s u c, 1}$ is given by the following equation.

$$
p_{s u c, 1}=\sum_{i=1}^{E[C W]} \frac{1}{E[C W]} \cdot\left(\frac{E[C W]-i}{E[C W]}\right)^{M-1}
$$

Furthermore, the summation of the probability of collision and the probability of deferring for one station is given by the following equation: $1-p_{\text {suc }, 1}=$ $p_{\text {col }, 1}+p_{\text {defer }, 1}$.

The contention window size for each contention procedure is increased by the increasing factor (IF) when a station experiences either a collision or a deferred situation, and goes to the minimum value with a successful packet transmission. Therefore, the average contention window size for each contention period is

$$
E[C W]=p_{s u c, 1} \times \min C W+\left(1-p_{s u c, 1}\right) \times E[C W] \times I F
$$

If we use the above equations (2) and (3), we can use an iterative process to obtain the average contention window size for each contention procedure $E[C W]$ and the probability, $p_{s u c, 1}$, of a successful packet transmission for one station. If the number of stations in the network is $M$, the total probability of successful packet transmission for the whole network is $p_{s u c, t o t a l}=p_{s u c, 1} \cdot M$ and the total average probability of collision for the whole network is $p_{\text {col,total }}=1-p_{\text {suc,total }}$. 


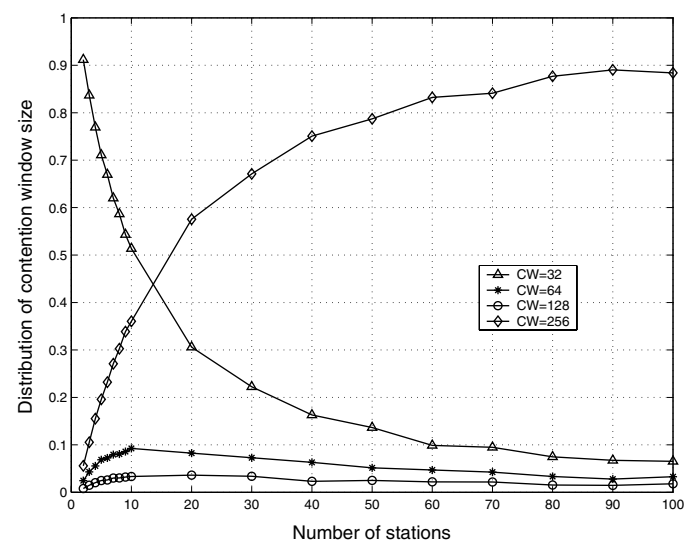

Fig. 1. Distribution for Contention Window Size

Now, we can calculate the average number of collisions for a successful packet transmission

$$
E\left[N_{c}\right]=\frac{p_{\text {col,total }}}{p_{\text {suc }, \text { total }}}
$$

To calculate the average idle backoff slot number $E[I d l e S l o t] / t_{\text {slot }}$, we need the probability of sending a packet at each contention window size $E[C W]_{P k S e n d}$. In Figure 1, an example of the distribution of contention window sizes for sending a packet in steady state is shown for the $\min C W=32, \max C W=256, I F=2$ case. In the 10 station case, $51 \%$ of stations have contention window size of sending a packet at $C W=32,9 \%$ of stations have $C W=64,3 \%$ of stations have $C W=128$, and $37 \%$ of stations have $C W=256$. As the number of stations increase, we can see the operational characteristics of the proposed algorithm follow those of the perfect scheduling. The average contention window size of sending a packet is

$$
\begin{aligned}
E[C W]_{\text {PkSend }}= & 32 \times p_{P k S e n d, 32}+64 \times p_{\text {PkSend }, 64}+128 \times p_{P k S e n d, 128} \\
& +256 \times p_{P k \text { Send }, 256}
\end{aligned}
$$

where $p_{P k S e n d, i}$ is the probability that a packet is transmitted with a contention window size $i$.

The average number of idle backoff slots is given by the following equation

$$
E[\text { IdleSlot }] / t_{\text {slot }}=\sum_{i=1}^{E[C W]_{P k S e n d}-1} i \times \frac{\left(E[C W]_{P k S e n d}-i\right)^{M-1}}{\left(E[C W]_{P k S e n d}\right)^{M}}
$$

In Figure 2, the throughput results of the proposed algorithm, the improved DCF (under the assumption that all stations have the same contention window range) and the IEEE802.11 MAC are shown for 10 and 50 contending stations, respectively. We can see that the throughput of the proposed MAC is significantly 


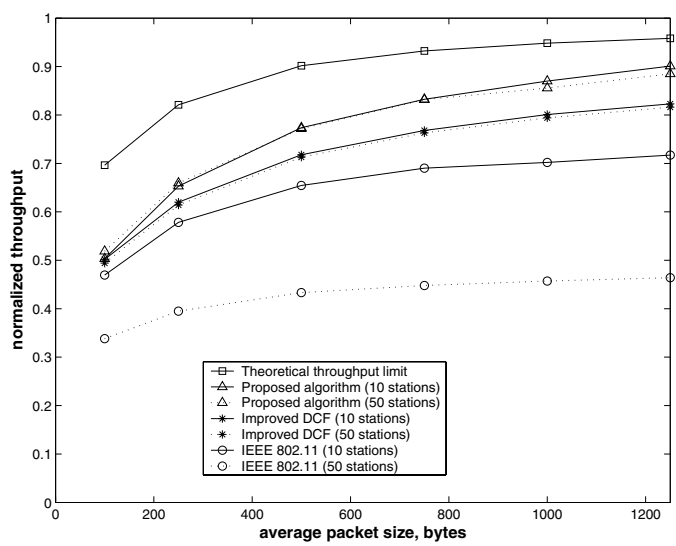

Fig. 2. Throughput results

improved compared with other MAC algorithms and close to the theoretical limit of perfect scheduling as the average packet size is increased.

\section{Conclusions}

The general assumption that all stations have the same contention window range in steady state results in sub-optimal solutions for performance analysis of distributed contention-based MAC algorithms because of its inherent limitations. We present a new assumption for the theoretical throughput limit from observing the operational characteristics of the perfect scheduling CSMA algorithm. The proposed algorithm based on the new assumption significantly improves the throughput performance and still provides easy implementation property in wireless networks. Extensive performance analysis and simulation studies for various performance factors have demonstrated that the proposed algorithm reduces the wasting overheads come from each contention procedure.

\section{References}

1. Bharghavan, V.: MACAW: A Media Access Protocol for Wireless LAN's. SIGCOMM'94, London, England, Aug. (1994) 212-225

2. Bianchi, G.: Performance Analysis of the IEEE802.11 Distributed Coordination Function. IEEE Journal on Selected Areas in Commun. 18 (2000) 535-547

3. Cali, F., Conti, M., Gregori, E.: Dynamin Tuning of the IEEE 802.11 Protocol to Achieve a Theoretical Throughput Limit. IEEE/ACM Trans. on Networking 8 (2000) 785-799

4. Aad, I., Castelluccia, C.: Differentiation mechanisms for IEEE 802.11. IEEE INFOCOM Anchorage, AK, USA (2001) 